\title{
Corrigendum
}

\section{Putrescine oxidase of Micrococcus rubens: primary structure and expression in Escherichia coli}

Hiroshi Ishizuka, SueHaru Horinouchi and Teruhiko BePPU

Journal of General Microbiology (1993), 139, 425-432

The title of the above paper was printed incorrectly. The correct title is shown above. 